\title{
Management of Retinal Detachment Associated with Morning Glory Disc Syndrome
}

\author{
Tyler Etheridge $^{a} \quad$ Zackery Oakey $^{b} \quad$ Michael M. Altaweel ${ }^{b}$ \\ aJohn A. Moran Eye Center, University of Utah, Salt Lake City, UT, USA; bepartment of \\ Ophthalmology and Visual Sciences, University of Wisconsin School of Medicine and Public \\ Health, Madison, WI, USA
}

\section{Keywords}

Morning glory disc anomaly $\cdot$ PAX6 gene mutation $\cdot$ Retinal detachment

\begin{abstract}
We report a case of morning glory disc anomaly in a young patient with tractional retinal detachment successfully repaired with complex pars plana vitrectomy, membrane peel, laser, and oil tamponade. A 19-year-old female with a history of right morning glory disc anomaly associated with PAX6 gene mutation presented with floaters, photopsia, central scotoma, and visual acuity (VA) of 1/200. A complex macula-involving tractional retinal detachment centered around the optic nerve with a morning glory disc anomaly. Retinal detachment was treated with 25-gauge pars plana vitrectomy with difficult separation of the posterior hyaloid. Fibrous preretinal membranes were peeled, a temporal relaxing retinotomy was required, subretinal fluid was drained through a superonasal retinotomy during air-fluid exchange, endolaser was applied, and tamponade was achieved with 1,000-centistoke silicone oil. The retina remained attached at 1 -year follow-up, with VA count fingers throughout. Morning glory disc is a rare congenital anomaly associated with PAX6 gene mutation that most often occurs unilaterally. It is rarely associated with tractional retinal detachment. Optimization of visual outcome is imperative despite a poor visual prognosis.
\end{abstract}

\section{Introduction}

Morning glory disc is a rare congenital anomaly characterized by an enlarged funnelshaped excavation in the optic disc, a ring of chorioretinal pigmentary changes that surround the excavation, and a central glial tuft that overlays the optic disc. Serous retinal detachment

\section{Karger $\stackrel{2}{\circ}$}


occurs in up to one-third of patients. We present a rare case of morning glory disc anomaly in a young patient with tractional retinal detachment successfully repaired with complex pars plana vitrectomy, membrane peel, laser, and oil tamponade.

\section{Case Report/Case Presentation}

A 19-year-old female with a history of right morning glory disc anomaly associated with a PAX6 gene mutation and myopia presented with a 1-month history of floaters, photopsia, and central scotoma. Her previous best-corrected visual acuity (VA) was 20/400 in the right eye and 20/20 in the left. She was otherwise healthy, including no congenital malformations or midline anomalies, was not on medications, and had no history of strabismus, ocular trauma, ocular surgery, or congenital glaucoma. On examination, VA was 1/200 OD and 20/20 OS. Intraocular pressure (IOP) was $18 \mathrm{~mm} \mathrm{Hg}$ OD and $11 \mathrm{~mm} \mathrm{Hg}$ OS. Examination demonstrated a complex macula-involving traction retinal detachment centered around the optic nerve with morning glory disc anomaly, with extension to the ora temporally. Despite the presence of vitreous hemorrhage, no retinal break was visualized with 360 scleral depressed examination (Fig. 1). The left eye was normal.

The patient underwent a 25-gauge pars plana vitrectomy with difficult separation of the posterior hyaloid from the elevated retina and optic nerve, requiring triamcinolone staining for visualization of the interface and bimanual elevation with a lighted pick and the vitrectomy cutter set on aspiration. There were no breaks noted intraoperatively; fibrous preretinal membranes were peeled, a temporal relaxing retinotomy was required, subretinal fluid was drained through a superonasal retinotomy during air-fluid exchange, endolaser was applied, and tamponade was achieved with 1,000-centistoke silicone oil. In the initial postoperative period, VA improved from hand motion to count fingers (CF), and IOP remained stable at $19 \mathrm{~mm} \mathrm{Hg}$. The retina remained attached at 1-year follow-up, with VA of CF and normal IOP throughout (Fig. 2, 3). Silicone oil emulsification developed at 18 months with a small amount passing into the anterior chamber, and posterior subcapsular cataract became more visually significant. The patient underwent 25 -gauge pars plana vitrectomy, oil removal, and cataract extraction with intraocular lens implantation 2 years after her initial operation. At her most recent follow-up, the patient's VA remains $\mathrm{CF}$.
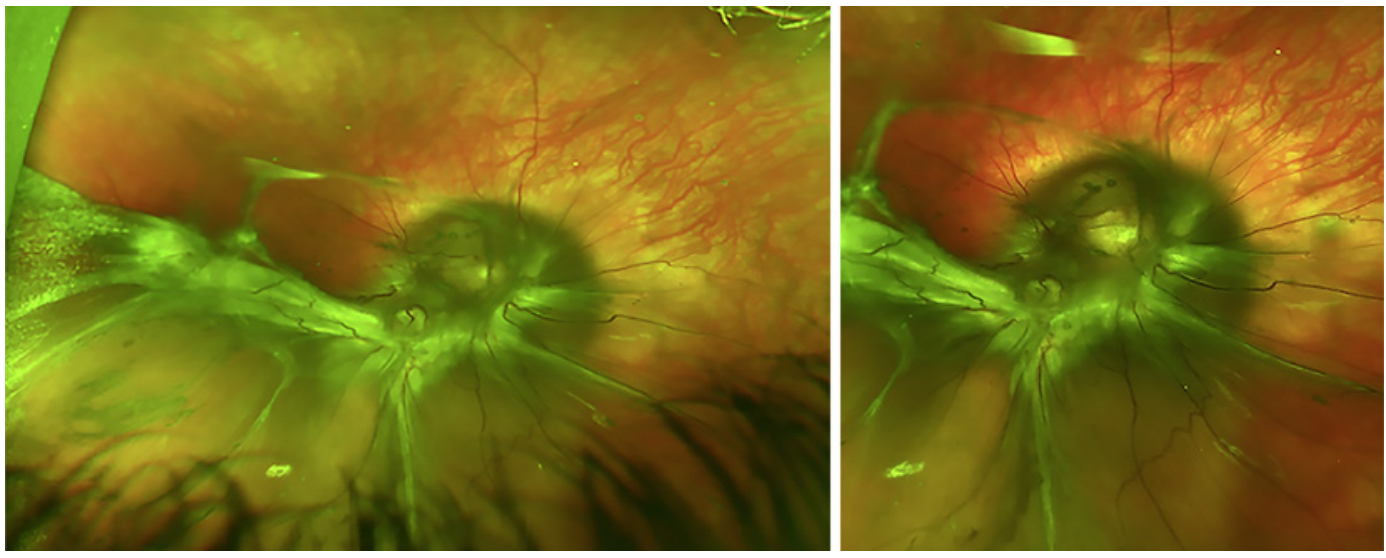

Fig. 1. Right eye at presentation. Note fibrosis, subretinal fluid, enlarged pigmented optic nerve consistent with morning glory disc anomaly, and tractional retinal detachment. 

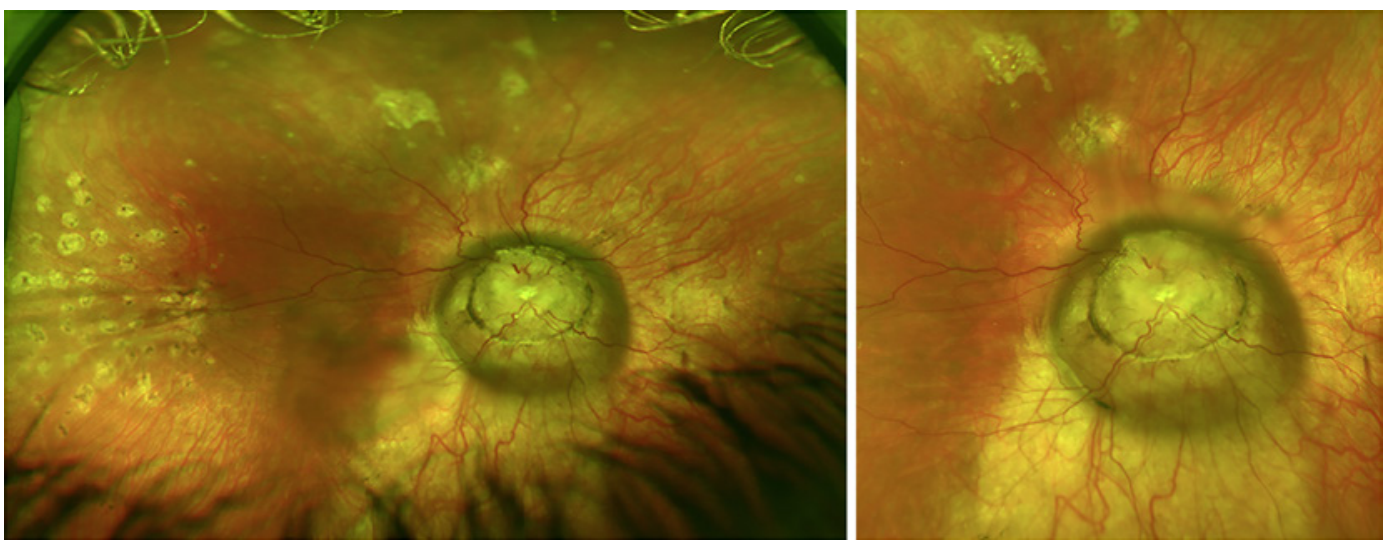

Fig. 2. Right eye at 1 -year follow-up. Note $360-^{\circ}$ flat peripheral retina, $360-^{\circ}$ endolaser, and flat temporal relaxing retinotomy.
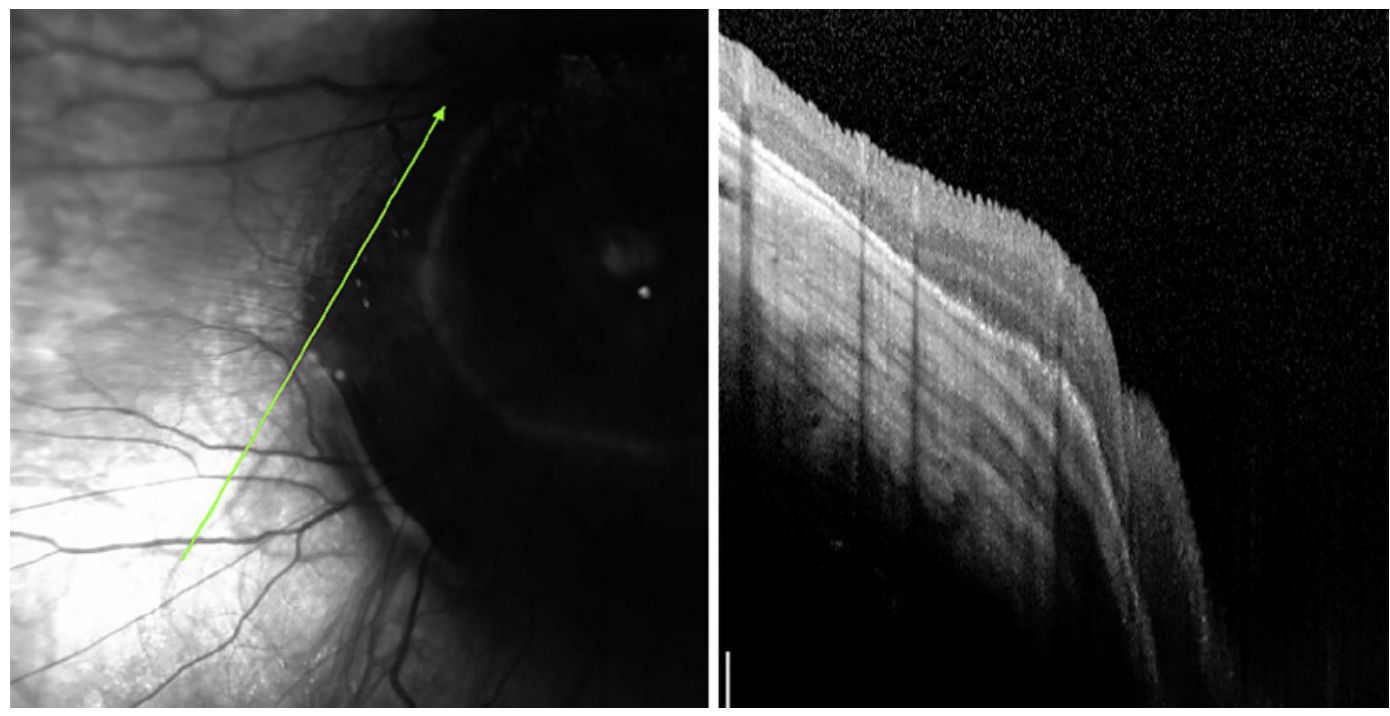

Fig. 3. Right eye OCT of repaired retinal detachment, fovea at edge of morning glory disc anomaly.

\section{Discussion/Conclusion}

Morning glory disc is a rare congenital anomaly first described by Kindler et al. [1]. The appearance was noted to resemble the morning glory flower. Three principal findings comprise the anomaly, including an enlarged funnel-shaped excavation in the optic disc, a ring of chorioretinal pigmentary changes that surround the excavation, and a central glial tuft that overlays the optic disc. The term "macular capture" applies if the excavation involves the macula [2]. The optic disc may appear elevated, and there may be an increased number of retinal vessels radiating from the disc, distorting the typical branching pattern. Not uncommonly, peripapillary arteriovenous communications and contractile movements within the anomaly corresponding to pulse pressure are present [3]. Although it can be bilateral [4], morning glory disc most commonly presents unilaterally, with similar rates of right or left eye involvement.

No clear etiology has been identified. However, morning glory disc may be due to a mesenchymal or fetal fissure closure abnormality [5]. The anomaly can be part of a syndrome, in which case the term "morning glory syndrome" is used. The most common associations include 
transsphenoidal basal encephalocele and midfacial malformations [6]. A basal encephalocele is a congenitally malformed outpouching of the meninges, which commonly contains the optic chiasm and hypothalamus that protrudes through a defect in the sphenoid bone. This malformation is often associated with other midfacial anomalies, including hypertelorism, wide nasal root, and cleft lip and palate. Another notable association is moyamoya syndrome, which is caused by progressive stenosis of the intracranial internal carotid arteries that leads to compensatory collateralization of vessels at the cranial base. On angiography, these collateral vessels produce the pathognomonic appearance termed "puff of smoke" [7]. Finally, neurofibromatosis type 2 and posterior fossa malformation, facial hemangioma, arterial anomalies, cardiac anomalies, and eye anomalies are also associated. Neuroimaging to evaluate for CNS involvement is required.

Morning glory anomaly may be confused with optic nerve coloboma and peripapillary staphyloma $[8,9]$. It is essential to differentiate optic nerve coloboma because of its association with coloboma of the eye, heart defects, choanal atresia, growth retardation, genitourinary abnormalities, and ear abnormalities syndrome. Optic nerve coloboma appears as an inferior white excavation, rather than pink or orange, that extends into the choroid and retina. It also lacks the central glial tuft and peripapillary pigmentation characteristic of morning glory anomaly. Peripapillary staphyloma also presents with optic disc excavation. However, it is differentiated by normal retinal vasculature and lack of a central glial tuft. Interestingly, morning glory disc anomaly is part of a clinically heterogeneous group of congenital cavitary optic disc anomalies including optic pit (Fig. 4), optic nerve coloboma, peripapillary staphyloma, and vacant optic disc (Fig. 5) [10]. Mutations in the PAX6 gene, which codes for a transcription factor

Fig. 4. Right eye optic nerve pit status post vitrectomy, fluid-gas exchange, and laser.

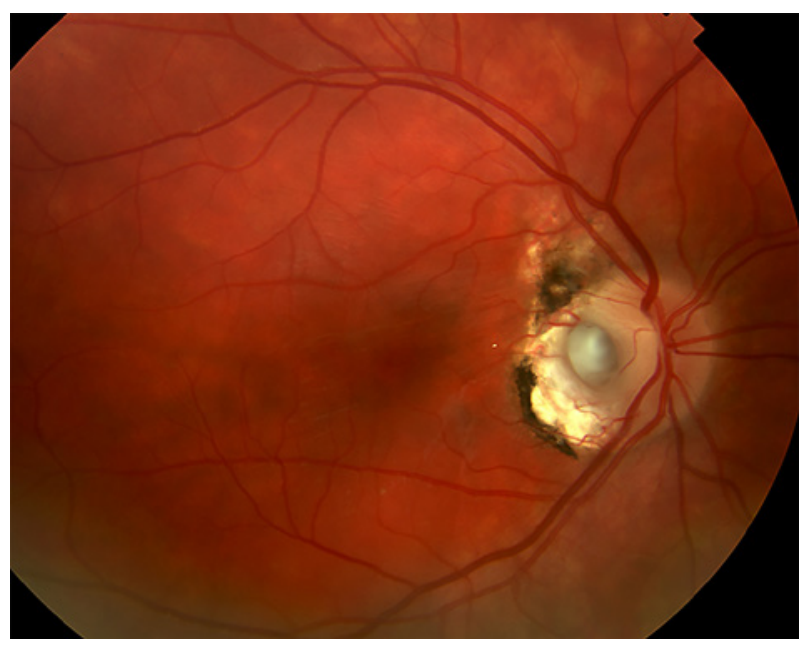

Fig. 5. Left eye vacant disc with coloboma and fibroglial tissue over the optic nerve.

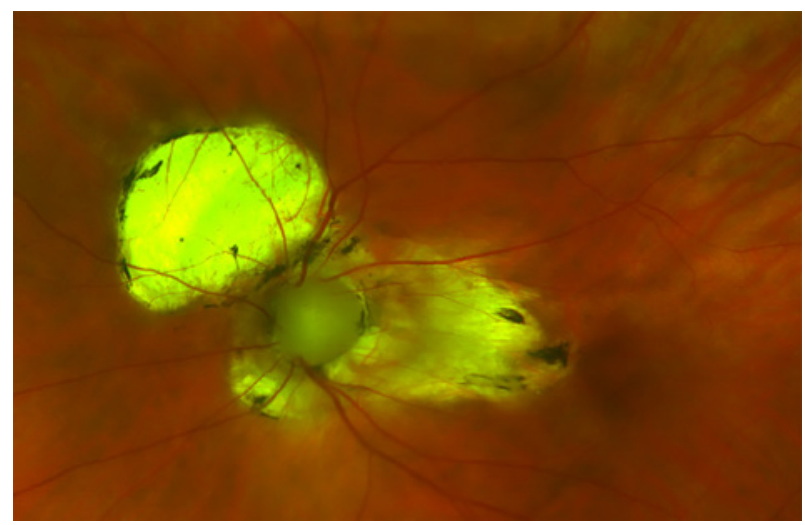


required for normal embryonic development of the eye, are most commonly associated with aniridia and Peter's anomaly [11]. However, as in our patient, PAX6 gene mutations have been implicated in several congenital cavitary optic disc anomalies, including morning glory disc [12].

Unfortunately, there is no treatment for morning glory disc anomaly, and the visual prognosis is poor, usually ranging from $20 / 100$ to $20 / 200$. Our patient's baseline VA was $20 / 400$ in the involved eye, which worsened with her complex retinal detachment. Morning glory disc anomaly is also associated with vision-threatening complications, the most common being serous retinal detachments (Fig. 6), which occurs in approximately 30\% of patients. Although the etiology linking serous retinal detachment and morning glory anomaly is unknown, it may be due to a communication between the subarachnoid and subretinal space within the excavation, which allows direct connection into the potential subretinal space [13]. Another proposed source of fluid is from the vitreous cavity through a slit-like retinal break at the edge of the excavated disc anomaly [14]. Traction on the retina likely contributes, either directly or by creating a secondary retinal break in the thin atrophic membrane surrounding the optic disc (Fig. 7) [15]. A series of optic disc coloboma and morning glory syndrome cases demonstrated a retinal break within a peripapillary defect in 5 of 6 cases. Retinal detachment was less likely to occur, remained subclinical, or spontaneously resolved in the absence of vitreous or glial tissue causing retinal traction within the anomaly. Removal of glial tissue may release tractional forces on the retina. The risk of causing another retinal break with glial tissue removal is high but may be reduced with laser endophotocoagulation at the defect margin to prevent passage of fluid into the subretinal space.

Our patient underwent a pars plana vitrectomy with membrane peel, endolaser, and silicone oil tamponade, which achieved good anatomical success. Review of other series
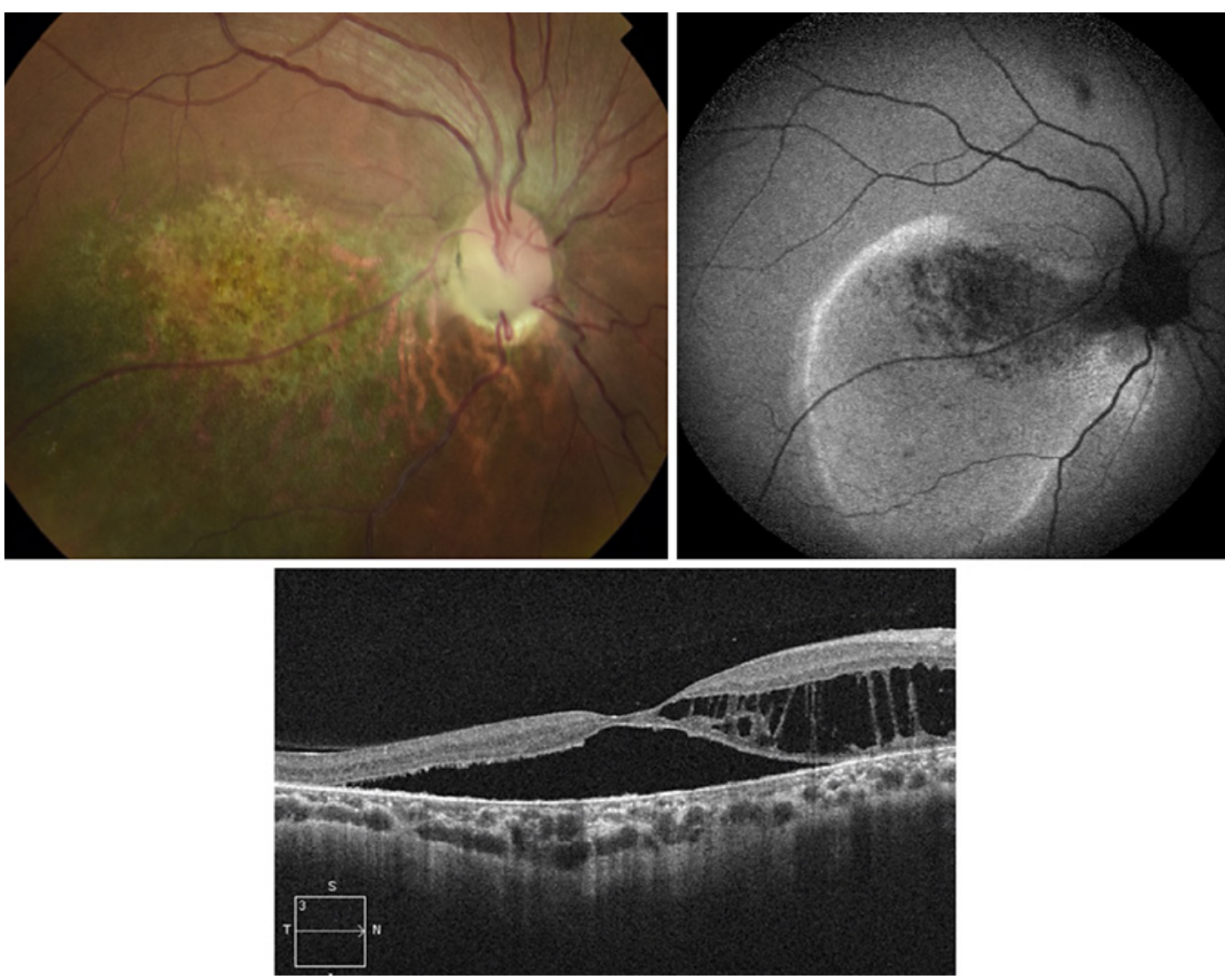

Fig. 6. Right eye morning glory disc anomaly with serous retinal detachment and retinoschisis shown on OCT.

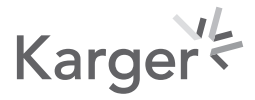



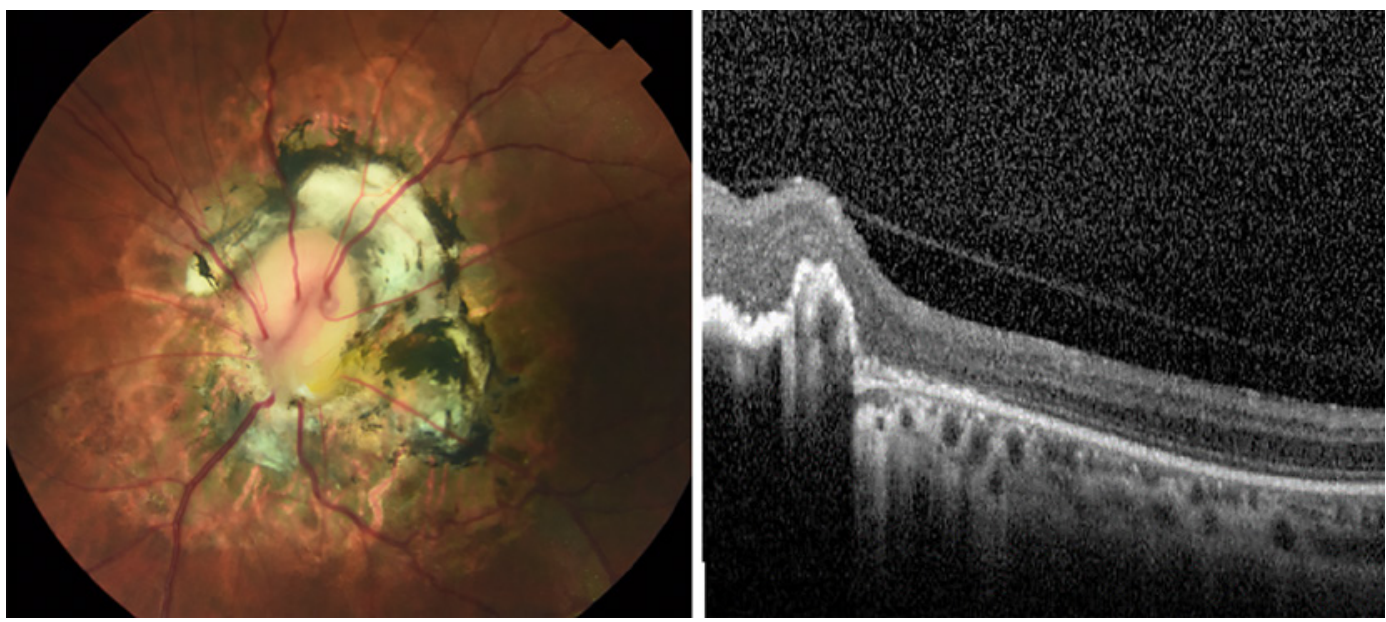

Fig. 7. Left eye morning glory disc anomaly with OCT showing surrounding thinned retina.

describing the surgical management of retinal detachment with morning glory disc anomaly demonstrates that vitrectomy with tamponade using long-acting gas or silicone oil provides the best anatomic and functional outcomes. The removal of fibroglial tissue causing traction is essential to prevent recurrent traction [15]. Also, the posterior hyaloid is strongly adherent due to the patient's young age and the fibrous adhesions present. Removal requires mechanical separation, which may be aided by staining the vitreous with triamcinolone, and elevating the hyaloid in a bimanual technique using a lighted pick and aspiration with the extrusion cannula or vitrectomy cutter [14].

Morning glory disc is a rare congenital anomaly of unknown etiology that most often occurs unilaterally. This anomaly may be rarely associated with traction retinal detachment. The detachment can be successfully repaired with pars plana vitrectomy and release of tractional elements with membrane peeling, elevation of the hyaloid, and relaxing retinotomy if required. Adherence of the retina is achieved with laser and oil tamponade. VA outcome is determined by the extent of the anomaly and whether the macula is involved. Optimization of visual outcome is imperative despite a guarded visual prognosis.

\section{Acknowledgements}

The manuscript does not include any nonauthor contributors to acknowledge.

\section{Statement of Ethics}

Written informed consent was obtained from the patient for publication of this case report and any accompanying images. The study adhered to the tenets of the Declaration of Helsinki. This study protocol was reviewed and the need for approval was waived by the University of Wisconsin Institutional Review Board.

\section{Conflict of Interest Statement}

The authors have no financial disclosures.

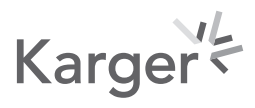


Etheridge et al.: Morning Glory Disc Syndrome

\section{Funding Sources}

Funding for this research was provided by an Unrestricted Grant from Research to Prevent Blindness, Inc. to the University of Wisconsin Department of Ophthalmology and Visual Sciences and National Eye Institute Grant U10EY014656 (Dr. Altaweel).

\section{Author Contributions}

Tyler Etheridge participated in the acquisition of data, interpretation of data, drafting of the manuscript, and critical revising of the manuscript. Zachary Oakey participated in the acquisition of data, interpretation of data, and critical revising of the manuscript. Michael M. Altaweel participated in the acquisition of data, interpretation of data, drafting of the manuscript, and critical revising of the manuscript. All the authors provided final approval of the manuscript version to be published.

\section{References}

1 Kindler P. Morning glory syndrome: unusual congenital optic disk anomaly. Am J Ophthalmol. 1970 Mar; 69(3):376-84.

2 Steinkuller PG. The morning glory disk anomaly: case report and literature review. J Pediatr Ophthalmol Strabismus. 1980 Mar-Apr;17(2):81-7.

3 Sawada Y, Fujiwara T, Yoshitomi T. Morning glory disc anomaly with contractile movements. Graefes Arch Clin Exp Ophthalmol. 2012 Nov;250(11):1693-5.

4 Beyer WB, Quencer RM, Osher RH. Morning glory syndrome. A functional analysis including fluorescein angiography, ultrasonography, and computerized tomography. Ophthalmology. 1982 Dec;89(12):1362-7.

5 Cennamo G, de Crecchio G, Iaccarino G, Forte R. Evaluation of morning glory syndrome with spectral optical coherence tomography and echography. Ophthalmology. 2010 Jun;117(6):1269-73.

6 Ellika S, Robson CD, Heidary G, Paldino MJ. Morning glory disc anomaly: characteristic MR imaging findings. AJNR Am J Neuroradiol. 2013 Oct;34(10):2010-4.

7 Komiyama M, Yasui T, Sakamoto H, Fujita K, Sato T, Ota M, et al. Basal meningoencephalocele, anomaly of optic disc and panhypopituitarism in association with moyamoya disease. Pediatr Neurosurg. 2000 Aug;33(2): 100-4.

8 Brodsky MC. Congenital optic disk anomalies. Surv Ophthalmol. 1994 Sep-0ct;39(2):89-112.

9 Lee BJ, Traboulsi EI. Update on the morning glory disc anomaly. Ophthalmic Genet. 2008 Jun;29(2):47-52.

10 Wang D, Pan X, Ji J, Gu S, Sun X, Jiang C, et al. A large family with inherited optic disc anomalies: a correlation between a new genetic locus and complex ocular phenotypes. Sci Rep. 2017;7(1):7799.

11 Prosser J, van Heyningen V. PAX6 mutations reviewed. Hum Mutat. 1998;11(2):93-108.

12 Azuma N, Yamaguchi Y, Handa H, Tadokoro K, Asaka A, Kawase E, et al. Mutations of the PAX6 gene detected in patients with a variety of optic-nerve malformations. Am J Hum Genet. 2003 Jun;72(6):1565-70.

13 Irvine AR, Crawford JB, Sullivan JH. The pathogenesis of retinal detachment with morning glory disc and optic pit. Retina. 1986;6(3):146-50.

14 Ho CL, Wei LC. Rhegmatogenous retinal detachment in morning glory syndrome pathogenesis and treatment. Int Ophthalmol. 2001;24(1):21-4.

15 Chang S, Gregory-Roberts E, Chen R. Retinal detachment associated with optic disc colobomas and morning glory syndrome. Eye. 2012 Apr;26(4):494-500. 